\title{
THE EXISTENCE OF A SOLUTION OF $f(x)=k x$ FOR A CONTINUOUS NOT NECESSARILY LINEAR OPERATOR
}

\section{S. VENKATESWARAN}

\begin{abstract}
It is proved that if $f$ is a continuous nonlinear operator on a Banach space $E$ then $f(x)=k x$ has a solution when $|k|$ is sufficiently large.
\end{abstract}

Introduction. Let $E$ be a Banach space and $f$ a continuous operator on $E$. That is, $f$ is a continuous, not necessarily linear mapping from $E$ into $E$. In this note we prove that $f(x)=k x$ has a solution when $|k|$ is sufficiently large.

THEOREM. Let $f$ be a continuous operator on a real Banach space E. Then, for each $\delta>0$, there exists $k_{\delta}>0$ such that whenever $|k| \geqq k_{\delta}$, there exists $x \in A_{\delta}$ satisfying the equation $f(x)=k x$, where $A_{\delta}=\{x:\|x\|<\delta\}$.

To prove the theorem we use the following fixed point theorem due to Altman [1, p. 97].

AltMAN FIXED POINT THEOREM. Let $A$ be a convex open bounded set in a Banach space $E$ containing 0 and let $\varphi: \bar{A} \rightarrow E$ be a continuous map satisfying $\|x-\varphi(x)\|^{2} \geqq\|\varphi(x)\|^{2}-\|x\|^{2}$ for all $x \in \partial A$. Then $\varphi$ has a fixed point.

Proof of THE THEOREM. Because $f$ is a continuous operator on $E, f$ is continuous at 0 . Hence there exists $\delta_{0}>0$ such that $\|f(x)\|<1+|f(0)|$ for $\|x\| \leqq \delta_{0}$. Let $\varepsilon=\operatorname{Min}\left(\delta, \delta_{0}\right)$. Let $A=A_{\varepsilon}$ where $A_{\varepsilon}=\{x:\|x\|<\varepsilon\}$. Choose $k_{\delta}=\varepsilon^{-1}(1+|f(0)|)$. If $|k| \geqq k_{\delta}$, then $|k| \geqq\|x\|^{-1} f(x)$, for all $x \in \partial A$. Therefore $\left\|k^{-1} f(x)\right\|^{2}-\|x\|^{2} \leqq\left(\|x\|-\left\|k^{-1} f(x)\right\|\right)^{2}$, for all $x \in \partial A$. But, by the triangle inequality, $\left(\|x\|-\left\|k^{-1} f(x)\right\|\right)^{2} \leqq\left\|x-k^{-1} f(x)\right\|^{2}$. Therefore, whenever $k \geqq k_{\delta}$ we have $\left\|k^{-1} f(x)\right\|^{2}-\|x\|^{2} \leqq\left\|x-k^{-1} f(x)\right\|^{2}$ for all $x \in \partial A$. Hence $k^{-1} f(x)$ satisfies the conditions of Altman's theorem. Therefore for $k \geqq k_{\delta}$, there exists $x \in A$ such that $f(x)=k x$. Since $A \subset A_{\delta}$ the theorem follows.

Received by the editors February 18, 1972.

AMS 1970 subject classifications. Primary 47H15; Secondary 47H10.

Key words and phrases. Banach space, nonlinear operator, fixed point theorem.

(c) American Mathematical Society 1972 
The following example shows that the conclusion of the theorem does not hold in an arbitrary normed linear space. Let $S$ be the set of all sequences $\left\{a_{n}\right\}$ of real numbers such that $a_{n}=0$ for all but a finite number of $n$ 's. Now $S$ is a normed linear space under the norm $\left\|\left\{a_{n}\right\}\right\|=\operatorname{Sup}\left|a_{n}\right|$. If we now define $f\left(\left\{a_{n}\right\}\right)=\left(1, a_{1}, a_{2}, \cdots\right)$, it is obvious that $f$ is continuous and that there does not exist $x \in S$ and $k$ real, such that $f(x)=k x$.

I wish to thank Professor L. A. Rubel for suggesting this problem.

\section{REFERENCES}

1. J. T. Schwartz, Non-linear functional analysis, Gordon and Breach, New York, 1969.

Department of Mathematics, University of Illinois, Urbana, Illinois 61801 